\title{
Intuitive reasoning about abstract and familiar physics problems
}

\author{
MARY KISTER KAISER \\ NASA Ames Research Center, Moffett Field, California \\ and \\ JOHN JONIDES and JOANNE ALEXANDER \\ University of Michigan, Ann Arbor, Michigan
}

\begin{abstract}
Previous research has demonstrated that many people have misconceptions about basic properties of motion. In two experiments, we examined whether people are more likely to produce dy. namically correct predictions about basic motion problems involving situations with which they are familiar, and whether solving such problems enhances performance on a subsequent abstract problem. In Experiment 1, college students were asked to predict the trajectories of objects exiting a curved tube. Subjects were more accurate on the familiar version of the problem, and there was no evidence of transfer to the abstract problem. In Experiment 2, two familiar problems were provided in an attempt to enhance subjects' tendency to extract the general structure of the problems. Once again, they gave more correct responses to the familiar problems but failed to generalize to the abstract problem. Formal physics training was associated with correct predictions for the abstract problem but was unrelated to performance on the familiar problems.
\end{abstract}

Recent studies have demonstrated that many adults hold erroneous beliefs concerning fundamental laws of motion (McCloskey, 1983; McCloskey, Caramazza, \& Green, 1980). For example, when asked to predict the trajectory of a ball exiting a curved tube, many college students respond that the ball will continue to curve, at least for some period of time. McCloskey and his colleagues explain such erroneous predictions as evidence that, when people are asked to reason abstractly about motion, their intuitive models frequently resemble a medieval impetus theory rather than a Newtonian model. The Newtonian model holds that, in the absence of external force, objects maintain a linear path. Alternatively, the impetus theory holds that setting an object in motion imparts to the object an internal energy, or impetus, that maintains the object's motion along its initial trajectory, be it linear or curvilinear.

Suppose people were asked to reason about problems that evoked actual motion events with which they are familiar. Would they make the same impetus-like error? Research in other cognitive domains raises the possibility that they would not: whereas many adults make errors in reasoning about abstract logic problems, performance is much better on problems that are logically

This research was supported in part by NIMH Training Grant T32MH16892 to the first author and in part by a grant from AFOSR to the second author. Portions of this paper were presented at the Annual Meeting of the Midwestern Psychological Association, Chicago, May, 1985.

We would like to thank Michelene Chi, Alice Healy, and Michael McCloskey for their helpful comments. Requests for reprints should be sent to Mary Kaiser, NASA Ames Research Center, Mail Stop 239-3, Moffett Field, OA 94035. equivalent to the abstract problems but that make reference to familiar situations. For example, in Wason's (1966) selection task, subjects were presented with cards showing a letter on one side and a number on the other. They were required to choose which cards needed to be examined to determine the validity of the statement: "If a card has an A on one side, then it has a 4 on the other." When presented four cards showing an "A," a "B," a " 4 ," and a "7," many subjects responded that the " $A$ " and " 4 " cards must be examined (instead of the logically correct " $A$ " and "7"), an error termed "affirming the consequent." Other logical errors, such as insisting that all cards must be examined, were also observed. This would suggest that adults make systematic errors in logical reasoning, much as McCloskey's work suggests that adults make systematic errors in mechanical reasoning.

However, research has shown that subjects can solve problems that are formally identical to the selection task if they are presented in realistic, thematic contexts (see Evans, 1982). Drawing on the now-defunct British postal rule requiring more postage for sealed than unsealed envelopes, Johnson-Laird, P. Legrenzi, and M. S. Legrenzi (1972) asked subjects to examine letters for violations of the rule, "If a letter is sealed, then it has a 5d stamp on it." This problem is formally identical to Wason's (1966) selection task. Older subjects, familiar with the postal system, performed far better on the envelope task than on Wason's task. Younger subjects who had no previous experience with the postal rule performed no better on the envelope task.

Our Experiment 1 is concerned with the possibility that familiarity may breed success: We examined whether or 
not people would give more accurate trajectory predictions on a somewhat familiar motion problem than on a more abstract version of the problem. In addition to examining performance on the two problems, we were interested in whether or not subjects would recognize the similarity between the problems and perform better on the abstract problem if it was presented after the familiar one.

\section{EXPERIMENT 1}

\section{Method}

Subjects. Eighty college students (40 males and 40 females) were recruited in the hallway of a classroom building at the University of Michigan. Half of the students ( 20 males and 20 females) had taken physics courses in either high school or college.

Materials and Procedures. A clear plastic spiral tube was mounted on a $60 \times 80 \mathrm{~cm}$ plywood board that lay flat on a level table. The tube was $2.2 \mathrm{~cm}$ in diameter and formed a spiral of $540^{\circ}$ rotation with an interior diameter of $25 \mathrm{~cm}$ (see Figure 1a). One end of the tube was elevated such that it appeared that a ball or liquid inserted in the elevated end would travel through the tube at a moderate speed. Half of the subjects were given the following instructions: "Suppose I take this ball bearing and place it in this (the elevated) end of the tube. It would roll around the tube and come out here (indicate mouth of tube). I'd like you to draw the path that the ball would take when it exited the tube." Once the subject had produced a response, a second problem was presented: "Okay, now imagine that we connect a hose to the elevated end of the tube and send water through it. The water would flow through the tube and come out here (at the mouth of the tube). Could you draw the path that the main part of the stream of water would take
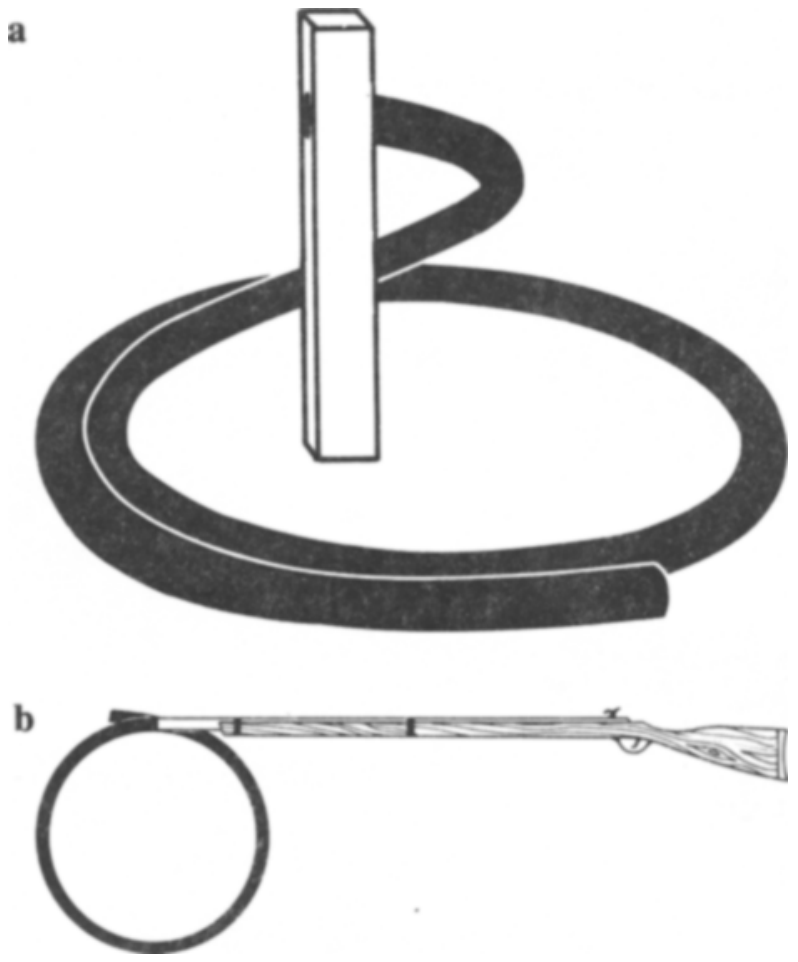

Figure 1. Schematics of apparatus employed in Experiment 1 (a) and Experiment 2 ( $a$ and $b$ ).

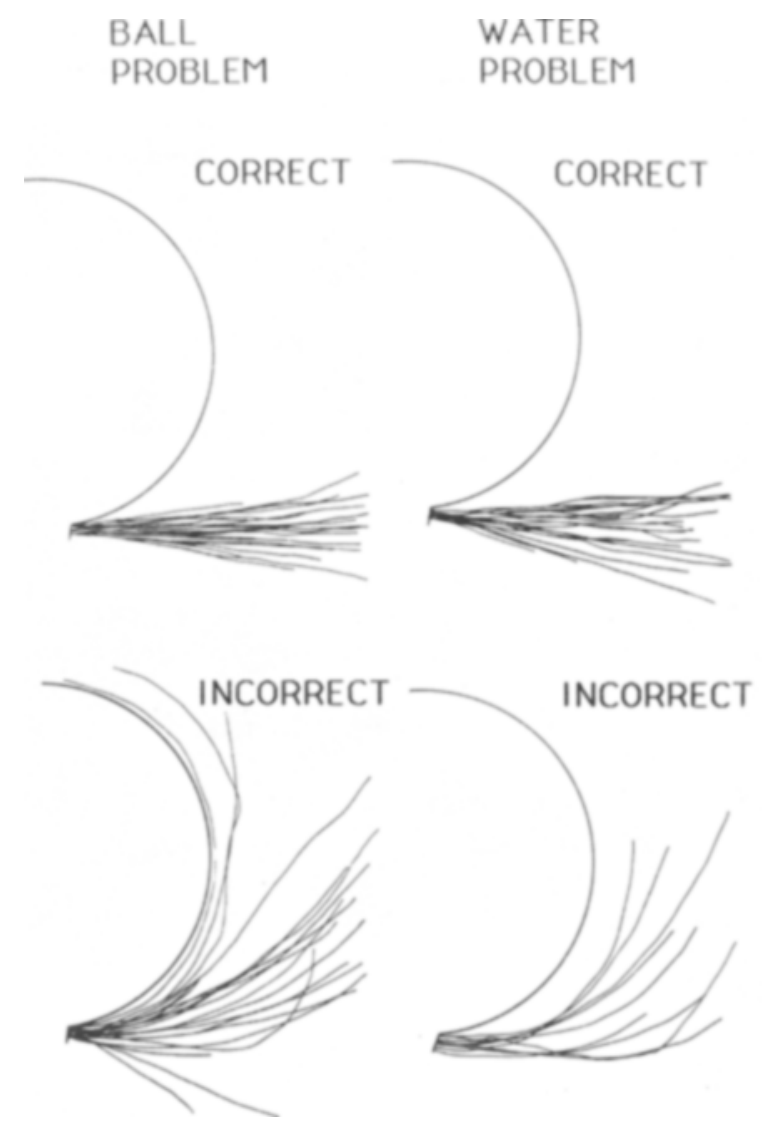

Figure 2. Examples of correct and incorrect responses on the Water and Ball problems in Experiment 1.

when it came out of the tube?" The other half of the subjects were administered the problems in the opposite order.

The ball problem has been used by a number of researchers to investigate people's understanding of curvilinear motion (e.g., McCloskey et al., 1980). Typically, a sizable proportion of subjects' answers include references to nonexistent forces and influences (e.g. , curvilinear momentum). The water problem was chosen since water shooting from a curved garden hose is a closely related event that is familiar to most people. Since most subjects have seen that the curvature of the hose does not affect the water's path, we hoped that people would draw upon this experience in solving the problem.

Subjects made their predictions by drawing a path on a $28 \times$ $55 \mathrm{~cm}$ piece of paper placed on the board at the mouth of the tube. Subjects were asked to describe verbally the path they drew to clarify any ambiguity. The experimenter recorded the subject's gender, and inquired about his or her coursework in physics. Subjects were thanked and paid for their participation.

\section{Results}

A response was coded as correct if the path arced no more than $10^{\circ}$ throughout its length and was tangent to the point of exit. Examples of correct and incorrect responses are illustrated in Figure 2. Subjects produced far more correct predictions for the water problem than for the ball problem. Fifty-three people ( 31 men and 22 women) drew linear paths for the water problem, compared with 31 subjects ( 22 men and 9 women) for the ball 
problem. This effect was highly significant $\left(\chi^{2}(1)=\right.$ $12.13, p<.005)$. Log-linear analyses demonstrated a strong gender effect for the ball problem $\left[\chi^{2}(1)=9.45\right.$, $p<.005]$ and a lesser gender effect for the water problem $\left[\chi^{2}(1)=4.59, p<.05\right]$. Physics training had a marginal effect on performance on the ball problem $\left[\chi^{2}(1)=\right.$ $2.93, p<.10$ ] but no significant effect on performance on the water problem $\left[\chi^{2}(1)=1.21\right]$.

To examine whether or not subjects transferred their correct solutions on the water problem to the more abstract ball problem, we tested for a problem-order effect among those subjects who answered the water problem correctly. Were subjects who demonstrated a correct understanding of the water problem more likely to answer the ball problem correctly if it was administered second? Of the 53 subjects who gave a correct response to the water problem, 27 were administered the ball problem first. Ten of these subjects (37\%) were correct on the ball problem. The other 26 subjects answered the ball problem after correctly answering the water problem. Fifteen of them $(58 \%)$ were correct on the ball problem. This difference is not significant $\left(\chi^{2}=2.26, p>.10\right)$. Thus, correctly solving a familiar problem on curvilinear motion immediately prior to attempting an abstract curvilinear problem did not significantly enhance performance on the latter.

\section{EXPERIMENT 2}

The lack of transfer in Experiment 1 is quite striking, especially since the abstract problem was administered immediately after the common-sense problem and even used the same apparatus. Why did subjects fail to reason analogically from the water problem to the ball problem? Is it possible to improve subjects' transfer by offering more than one common-sense exemplar? These questions were addressed in Experiment 2.

The literature on analogical problem solving suggests that a critical obstacle to solution transfer is the failure to recognize the relevant similarities among problems (D. Gentner \& D. R. Gentner, 1983; Holyoak, 1984). Providing multiple exemplars of a solution type often enhances people's ability to recognize abstract similarities (Holyoak, 1984). We investigated whether providing a second common-sense motion problem increased the likelihood of solution transfer to the abstract problem. Furthermore, we asked subjects to justify their predictions, particularly when their solutions for the two types of problems differed.

\section{Method}

Subjects. Eighty-one University of Michigan female students participated in the experiment. Forty-three of them had taken physics courses in either high school or college. Subjects were recruited from a subject pool at the university and were paid for their participation.

Materials and Procedure. Subjects were administered one abstract and two familiar curvilinear motion problems. The ball problem from Experiment 1 was the abstract problem. One of the familiar problems was the water problem of Experiment 1 . The other problem required subjects to predict the path of a bullet when fired from a rifle with a curved barrel. The rifle was oriented such that the curved barrel lay flat on a horizontal surface, similar to the curved-tube apparatus. A pilot study indicated that, although people had never used such a weapon, many insisted that they had seen them used (in cartoons and movies) and professed an intuitive understanding that bullets would have to travel straight regardless of the shape of the barrel. The experimental apparatus is schematized in Figures $1 a$ and $1 b$.

Subjects were instructed to draw the path of the ball, the water, or the bullet upon exiting the curved tube or barrel. Forty of the subjects were given the two familiar problems (water and rifle) first, followed by the abstract (ball) problem. The other subjects were administered the abstract problem first. The order of presentation for the two familiar problems was counterbalanced across subjects. After they had made all three predictions, 41 of the subjects (every other subject) were asked to explain their predictions and to justify any discrepancies in the paths.

\section{Results}

Subjects' responses were coded as in Experiment 1. The patterns of subjects' predictions are shown in Table 1. It should be noted that a fairly conservative classification scheme was used for the familiar problems: Subjects had to produce correct predictions for both familiar problems to be classified as accurate on that problem type. Very few subjects (12) gave incorrect predictions for both familiar problems.

Even with such a classification scheme, subjects were found to give more accurate predictions for the familiar problems than for the abstract problem $\left[\chi^{2}(1)=4.24\right.$, $p<.05]$. As in Experiment 1, there was no evidence of transfer of correct solutions from the familiar to the $\mathrm{ab}$ stract problems $\left[\chi^{2}(1)=1.29\right]$. Having correctly solved

Table 1

Patterns of Subjects' Responses for Abstract and Common-Sense Curvilinear Motion Problems (Experiment 2)

\begin{tabular}{lccc}
\hline & \multicolumn{2}{c}{ Number of Subjects } \\
\cline { 2 - 4 } Pattern of Responses & Common-Sense Problems First & Abstract Problem First & Total \\
\hline $\begin{array}{l}\text { Accurate Predictions for all Problems } \\
\text { More Accurate Predictions for Common- }\end{array}$ & $11(6)$ & $17(14)$ & $28(20)$ \\
$\quad$ Sense Problems & $10(7)$ & $13(5)$ & $23(12)$ \\
$\begin{array}{l}\text { More Accurate Prediction for Abstract } \\
\quad \text { Problem }\end{array}$ & $8(2)$ & $3(2)$ & $11(4)$ \\
$\begin{array}{l}\text { Inaccurate Predictions for Common-Sense } \\
\text { and Abstract Problems }\end{array}$ & $13(3)$ & $6(4)$ & $19(7)$ \\
\hline
\end{tabular}

Note-Numbers in parentheses indicate number of subjects with formal physics training. 
two familiar curvilinear problems did not enhance subjects' performance on the abstract problem.

How did subjects who gave correct responses for the familiar problems justify their erroneous responses to the ball problem? Examination of these subjects' protocols indicated three basic justifications: First, in the case of the rifle, subjects noted that the barrel had less curvature $\left(360^{\circ}\right)$ than did the ball apparatus $\left(540^{\circ}\right)$. Second, in the case of the water, subjects contended that liquids and solids had different motion properties; water would not acquire curvilinear "momentum" the way a ball would. Finally, subjects cited several irrelevant dynamic properties, notably speed, pressure, and weight. Speed was most often seen as a determining factor. Subjects reported that since the bullet (and sometimes the water) would travel faster than the ball, there would be less tendency for the bullet (and water) to "pick up" curvature from the tube. Two examples of subjects' protocols are given in the Appendix. Both of these subjects gave correct responses to the two common-sense problems but curvilinear responses to the abstract problem.

Most subjects who gave correct responses to all three problems noted the underlying similarity. Newton's first law of motion (or some more vernacular version) was often cited, as was the tendency of objects to move in a "natural" path (i.e., a straight line). The remainder of these subjects either were unable to justify their correct responses or acknowledged that they were just guessing.

Experience-based explanations were given for all problems, although more were given for the familiar problems than for the ball problem. Interestingly, incorrect responses were sometimes justified with inappropriate experiences, such as citing whirlpools as a basis for a curvilinear path for water.

\section{GENERAL DISCUSSION}

The data from both experiments indicate that people give more accurate predictions to some curvilinear motion problems than to others. Furthermore, a correct solution on one type of problem is not generalized to another, usually because irrelevant differences are noted in the problems.

One model that is generally consistent with the data holds that subjects apply formal physical principles to both kinds of problems, but that their principles are inaccurate and include such irrelevant factors as the object's velocity and the amount of tube curvature. Certainly, protocols indicate that subjects employ a number of inappropriate physical properties in their justifications. Subjects' allusions to specific experiences may merely reflect an attempt to provide concrete examples of their basic, abstract beliefs. However, if such a model is correct, it is not clear why these misconceptions should impact on subjects' ball predictions more often than on their water or rifle predictions. It would be rather serendipitous that the familiar problems we selected tapped more accurate formal physical principles than did the abstract problem we chose.
An alternative interpretation of the data is that people are able to reason more appropriately about motion problems when they are related to specific, concrete, familiar experiences. The facilitation of reasoning that results from placing an abstract problem in a familiar context has been examined in other cognitive domains, notably in the area of deductive reasoning. Context effects, or "facilitation by realism," have been found to affect performance on syllogistic reasoning (Wilkins, 1928) as well as on a number of variations of the Wason selection task (see Evans, 1982, for a summary). As in our motion problems, however, the facilitation did not generalize to subsequent abstract problems.

Evans (1982) has discussed context effects and the lack of solution transfer from the familiar to the abstract deductive reasoning problem. He argues that the studies that demonstrate the greatest context effects (e.g., JohnsonLaird et al., 1972) may present problems that are too realistic. That is, they do not require subjects to reason at all, but rather allow for a solution based on specific experiences. Such a model would certainly explain the lack of transfer: the relevant similarity in the problems is not recognized since the familiar problem is never processed in formal terms.

We propose a similar model for our subjects' performance. Subjects draw on specific experiences to solve the common-sense problems, and need not employ formal reasoning. The abstract problem, evoking no specific memory, requires subjects to draw upon their formal understanding of physics (which is often erroneous). Thus, we suggest that subjects apply a two-stage approach to solving these problems. First, they search for a specific solution based on relevant experiences. If this search fails, they default to a reasoning process employing formal understanding of mechanics. Such default reasoning models have been proposed in many areas of cognitive psychology (e.g., Siegler, 1981) and artificial intelligence (e.g. Reiter, 1980).

The striking lack of transfer can also be accounted for by such a model. Reasoning by analogy is dependent upon recognizing the relevant similarities of the base problem and the target problem (D. Gentner, 1982). Since the relevant similarities of the common-sense and abstract problems exist only at the level of formal analysis, it is necessary that the common-sense problems be viewed in formal terms for transfer to occur. However, subjects' protocols suggest that most people are able to map the common-sense problem to experience-based solutions on a very concrete level. Since the common-sense problems are only considered on a concrete level, similarity recognition is not possible. The use of two common-sense problems in our Experiment 2 did not improve subjects' solution transfer, although other researchers have found the provision of multiple exemplars to enhance subjects' awareness of formal similarities among problems (Holyoak, 1984).

Finally, our model explains why people demonstrate inconsistency in their reasoning concerning motion 
problems. Many people who give impetus-type responses to one problem will provide a correct prediction on the next. We suggest that this is because people do not always draw upon their formal respresentation of physics, but only do so when they are unable to find an acceptable solution based on specific experiences in their memories. The problem of deciding what is or is not a relevant experience is still an issue, but our main point is that people draw on their formal models only after such a solutionby-analogy method fails. In the problems we employed, subjects usually failed to find a relevant experience for the abstract problem and dismissed the common-sense problems as irrelevant based on extraneous factors (e.g., velocity, substance). What we propose, therefore, is that reasoning by analogy is the default strategy most people apply to motion problems (particularly if they lack formal physics training). Only when people are unable to map the target problem to an appropriate base do they draw upon formal representations. It is then that errors reflecting an impetus model of physics emerge from many individuals.

\section{REFERENCES}

Evans, J. ST. B. T. (1982). The psychology of deductive reasoning. London: Routledge \& Kegan Paul.

GENTNER, D. (1982). Are scientific analogies metaphors? In D. S. Miall (Ed.), Metaphor: Problems and perspectives (pp. 106-132). Brighton, Sussex, England: Harvester Press.

Gentner, D., \& Gentner, D. R. (1983). Flowing waters or teeming crowds: Mental models of electricity. In D. Gentner \& A. Steven (Eds.), Mental models (pp. 99-130). Hillsdale, NJ: Erlbaum.

Holyoak, K. J. (1984). Analogical thinking and human intelligence. In R. J. Sternberg (Ed.), Advances in the psychology of human intelligence (Vol. 2, pp. 199-230). Hillsdale, NJ: Erlbaum.

Johnson-Laird, P. N., Legrenzi, P., \& Legrenzi, M. S. (1972). Reasoning and a sense of reality. British Journal of Psychology, 63, $395-400$.

McCloskey, M. (1983). Intuitive physics. Scientific American, 248(4), 122-130.

McCloskey, M., Caramazza, A., \& Green, B. (1980). Curvilinear motion in the absence of external forces: Naive beliefs about the motion of objects. Science, 210, 1139-1141.

REITER, R. (1980). A logic for default reasoning. Artificial Intelligence, $13,81-132$.

SIEGLER, R. (1981). Developmental sequences within and between concepts. Monographs of the Society for Research in Child Development, 46(2).

Wason, P. C. (1966). Reasoning. In B. M. Foss (Ed.), New horizons in psychology I (pp. 135-151). Harmondswoth, England: Penguin.

WILKINS, M. C. (1928). The effect of changed material on the ability to do formal syllogistic reasoning. Archives of Psychology (New York) No. 102.

\section{APPENDIX \\ Sample Subject Protocols from Experiment 2}

Subject 20

Q: Could you explain for each of the situations why you thought the objects took the paths you drew?

A: I guess when I think in terms of bullets, bullets always go straight; when it came out of the gun, I figure it would go straight. And the water, water also seems to go straight out, no matter how much like a garden hose is twisted around, so I imagine the water coming out straight. The ball, I imagine, staying next to the tube, like just following around the tube.

Q: Could you tell me how the speed of the object affects the path it takes?

A: If it were going really fast, it would go straighter than if it were going slow.

Q: Why is that?

A: When you roll a ball very slowly, it tends to go off to the side, where if you throw it faster, it takes a straighter path. I guess maybe it's the gravity behind it.... well, it's not even gravity, I guess; it's more like inertia, or the energy it has.

Q: Could you tell me your background in physics?

A: Two years in high school. I got As.

Subject 21

Q: Could you explain why you thought the objects took the paths you drew?

A: I'm going to assume that the ball's going to come out slower here, and because it's been going around and not going real fast, it's going to take the path it had, and curve around. With the gun, the curve was less on the tube, and there's more force, so it's going to be going more straight forward. I don't known the physics behind it, but since it hadn't been going real slow, it's going to take the most direct path, which will be straight. With the water, it's the same reasoning as with the shotgun, it's coming out faster, so it's going to take the direct path, and that's more out straight.

Q: How does the speed of the object affect the path it takes?

A: The faster the object goes, the straighter or more direct path it will take.

Q: Why is that? What about its going faster makes it take a straighter path?

A. Gravity's going to want to hold it in more to the circular path, but when it's going faster, then you get away from the effects of gravity.

Q: What's your physics background?

A: Very limited. Just reading on my own, and what they threw in chemistry....I got an A in chemistry.

(Manuscript received August 21, 1985; revision accepted for publication Febnuary 10, 1986.) 